\title{
PSYCHIC STRESS IN C HEPATITIS VIRUS INFECTION
}

\author{
Camelia Patrascu Boanca ${ }^{1}$, Mihaela Minulescu ${ }^{2}$, George Gherlan ${ }^{3}$, Petre Iacob Calistru ${ }^{4}$ \\ 1 "Victor Babes" Diagnostic and Treatment Center, Bucharest \\ ${ }^{2}$ Romanian Association for Analytical Psychology, Bucharest \\ 3 "Victor Babes" Diagnostic and Treatment Center, Bucharest \\ 4 "Carol Davila" University of Medicine and Pharmacy, Bucharest
}

\begin{abstract}
The purpose of this review is to examine if the psychic stress can operate as a co-factor in the pathogenesis of the infection with $\mathrm{C}$ virus hepatitis. $\mathrm{C}$ virus hepatitis agents represent a common environmental stressor and the human body reaction to the stressor can be regarded as a stress too. Patients have experienced and reported various emotional reactions after being diagnosed with chronic HCV. The intensity of emotional reaction varies from one patient to another, and has been differently experienced and reported.

The vast majority of patients experience a decreased health related quality of life (HRQOL). The most common psychological reactions are fatigue, depression, fear, anxiety, anger, negative self esteem, stigma and sadness.

Depression appears to be present in those with HCV infection in a higher percentage than it is in the general population.

On many instances, the emotional reactions appear to be influenced by the level of education, geographical area, cultural background, awareness of the disease, gender, age, local environment and culture, wealth, time of diagnose and treatment. Various studies and researches lead us to believe that an improvement in depressive symptoms lead to an improvement in fatigue and well being.

Keywords: depression in HCV patients, quality of life in HCV patients, HCV patients social
\end{abstract} status

\section{INTRODUCTION}

Liver failure may lead to insufficient intake, nutrition malabsorption and massive protein consumption, which in turn can cause a negative nitrogen balance.

Stressors have been given different meanings over time, but probably the simple one is that they represent internal or external challenges that disrupt the normal internal environment. Humans developed various mechanisms intended to restore homeostasis in response to stressors. The two main systems that deal with the stressors are: a) the hypothalamic-pituitary-adrenal (HPA) axis and b) sympathetic nervous system (SNS).

"General stress" (1), as defined by A.W. von Eiff, is a "psycho-physical reaction of the human body caused by stressors that operate on the brain, triggering, due to the cortico-limbic connections with the hypothalamus, a string of neuro-vegetative and endocrine reactions, with repercussions all over the body".

Most of the HCV infected patients report various symptoms, like fatigue, depression, anxiety, irritability, headaches, myalgia, isolation and anorexia. Most of those symptoms are associated with an impaired quality of life and psychological disturbances.

Fatigue has been reported to be one of the major and the most frequent symptom of hepatitis $\mathrm{C}$ virus (HCV). The symptom was reported by almost one half of the chronically infected patients.

Depression is the second most common factor that influence health related quality of life (HRQOL).

Often, people are unaware as to how to prevent the transmission of HCV. Patients reported stories of family and friends that kept them away from their children. 


\section{DISCUSSION}

A study conducted on 642 patients (2) concluded that patients with hepatitis $\mathrm{C}$ with or without cirrhosis have significantly reduced their health related quality of life. The study infers that the perceived status of the subjects who had a successful response to interferon therapy improved their health related quality of life.

Another study (3) of HCV infected patients without cirrhosis were assessed for their health related quality of life using the short SF36 symptomatology questionnaire. Although the subjects with chronic HCV who used intravenous drugs had a very low health related quality of life (HRQOL), the subjects who had never used drugs also exhibited low values of the health related quality of life.

Since the early 1960 's, it was quite clear for the doctors and science that the central nervous system (CNS) and the immune system (IS) are liked. Dr. E.L. Cooper (4) was explaining that both systems have the function of memory and learn by experience. Immunization or prior experience means that the author acquired tolerance or hypersensitivity. Being hypersensitive means that one can react excessively because of similar prior traumatic experience.

There were reported different reactions of the $\mathrm{HCV}$ infected patients dependent on personal traits of the patients. The defensive patients are those that will be depressed, isolated, careless, anxious and culpable. The other group of patients is the aggressive one, which is seeking revenge and are antisocial (5).

Most of the time, one's effort to manage stressful experiences lead to engaging in a behavior (alcohol, sleep disorder, etc.) that could modify the immune system processes (6). This behavior represents a potentially important pathway linking stress to the immune system.

In a 2001 study (7), 84 participants were administered 3 hepatitis B vaccinations. Five months after the first dose and shortly after the second one, each participant was asked to complete a test and was taken a blood sample. Higher scores on a measure of dispositional positive affect were associated with a greater antibody response to vaccination. The study revealed that lower antibody response was predicted independently by (a) high trait negative affect and (b) diminished T-cell proliferation in response to PHA. These data provide evidence that a negative affect and the magnitude of stress-induced suppression of immune function may have clinical significance.

In 2011 (8), there were approximately 3 million people infected with chronic hepatitis $\mathrm{C}$ virus in the
United States. Many ot then had a high prevalence of substance abuse, neuropsychiatric effects and premorbid psychopathology caused by the hepatitis C treatment. Due to late identification of the disease and inefficient or non treatment of the psychiatric effects, a high morbidity and mortality in $\mathrm{HCV}$ patients is registered. For a successful treatment, an early identification of the disease and a prompt hepatitis $\mathrm{C}$ virus treatment should be accompanied by an appropriate psychiatric treatment. Patients with chronic HCV reported a large number of psychiatric conditions like fatigue, deficiencies in attention, deficiency in learning ability and memory, all these leading in the end to a reduced quality of life. These findings (9) have been found to be independent of the degree of the liver disease.

The Table (10) below shows the rates of psychiatric disorder patients with untreated hepatitis $\mathrm{C}$ virus.

\begin{tabular}{|l|c|c|}
\hline Disorder(s) & Current rate & Lifetime rate \\
\hline Mood disorders & $34 \%$ to $35 \%$ & $67 \%$ \\
\hline Major depressive disorder & $22 \%$ to $28 \%$ & $42 \%$ \\
\hline Anxiety disorders & $26 \%$ to $44 \%$ & $63 \%$ \\
\hline $\begin{array}{l}\text { Antisocial personality } \\
\text { disorder }\end{array}$ & $\begin{array}{c}\text { No rates; lifetime } \\
\text { diagnosis }\end{array}$ & $16 \%$ to $40 \%$ \\
\hline Psychotic disorders & $9 \%$ to $17 \%$ & $11 \%$ \\
\hline Substance use disorder & $56 \%$ & $56 \%$ to $86 \%$ \\
\hline
\end{tabular}

A study (11) performed at the Medical Faculty of Belgrade infers that social demographics play a role in chronic liver diseases. The patients with CLD - B are older and married as compared with patients with CLD $-\mathrm{C}$. The last category seems to be influenced by individuals behaviour mainly related to intravenous drug abuse as the route for $\mathrm{HCV}$ transmission. An increase of the cognitive dysfunction, depression and fatigue was reported in relation with $\mathrm{HCV}$ replication in the central nervous system.

In another study (12), patients with HCV reported that 5 out of 8 SF-36 domains, physical functioning, role-physical, general health, vitality, and social functioning were significantly lower compared with matched population controls.

However, the results on HRQOL do not seem to be uniform. American blood (13) donors patients with an incidence of fatigue of $61 \%$ and headache $54 \%$ were found to be HCV infected. However, fatigue rate was also present in $70 \%$ of the non $\mathrm{HCV}$, healthy blood donors suggesting no difference between the two groups. 
Although most of the studies conclude that HCV directly impairs the HRQOL, there may be other aspects like ethnic background, childhood instruction and treatment, personality traits etc. that may directly influence the HRQOL of the patients. A study carried on in Japan (14) found no characteristic subjective symptoms in patients with $\mathrm{HCV}$ compared with healthy controls, except a lower aggression score. This may be due to the high education and uniform cultural level of the Japanese society. Education is another factor that may influence HRQOL in HCV patients. Patients from Saudi Arabia (15) with lower education status reported lower scores than patients with higher education status, the possible cause being that these patients have limited understanding of $\mathrm{HCV}$ and $\mathrm{HBV}$, which in turn may lead to poorer disease management. In Egypt (16), another research reveals that patients who were unaware of their viral status reported no significant decrease in their HRQOL status compared to the rest of population. The same study infers that similar HRQOL may also be explained due to a possibly lower morbidity among Egyptian HCV-infected patients, by a higher morbidity among uninfected controls or by the effect of not knowing the diagnosis at the time of testing.

Depression and anxiety (17) have high prevalence in hepatitis $C$, and are largely undetected and treated. However, depression appears to be associated with adverse experiences of illness and not anxiety.

Assessment (18) of the severity of depressive symptoms in chronic HCV patients revealed that disability and fatigue are more closely related to depression severity than to hepatic disease severity. Antidepressant treatment trials in patients with $\mathrm{HCV}$ are recommended to determine whether an improvement in depressive symptoms lead to an improvement in fatigue and functioning.

Depressive symptoms typically begin early in the treatment, usually after $10-12$ weeks. There have been identified two distinct symptom clusters (19): a) a neurovegetative cluster, characterized by reduced energy, anorexia and psychomotor retardation, which typically begins within the first few months of treatment; b) few months later, a depression-specific syndrome appears. It includes depressed mood, anxiety and cognitive impairment.

The patients with chronic hepatitis $\mathrm{C}$ virus (HCV) infection experience increased levels of depression. Among (20) the etiological mechanisms assumed to be involved are premorbid psychiatric disease, genetic disposition to affective disorders, socio-economic factors, stigmatization and possi- bly HCV neuroinfection. Evidence to support that former intravenous drug abuse increases the risk of depression is lacking. It is particularly important to diagnose and treat depression in HCV patients, as it constitutes a relative contraindication to antiviral treatment and may jeopardize therapeutic outcome.

Depression (21) is prevalent in a considerable way in patients with HCV and it is escalated in the group of patients treated with interferon (G1). The presence of depression may be related to a poor treatment outcome. Psychiatric assessment and early intervention is mandatory in this population.

Regarding the levels of depression in HCV infected patients, there are some opinions suggesting that the depression rate is overestimated. This is mainly due to the fact that somatic symptoms of chronic illness and depression often overlap. Some researchers (22) have suggested that focusing on questions targeting cognitive and affective symptoms rather than somatic ones may be a more valid measure of depression in patients undergoing immunotherapy for $\mathrm{HCV}$.

A study conducted by Lehman C (23) on 120 patients (veterans) with chronic HCV established that they present high levels of depression (44.2\%), anxiety $(38.1 \%)$, post-traumatic stress disorder $(20.8 \%)$ and alcohol related problems (26.7\%). Also, depressive symptoms were also correlated with anxiety. The study concluded that psychiatric comorbidities were very common among veterans with chronic hepatitis $\mathrm{C}$ and correlated poorly with the diagnoses documented in the medical record.

Higher scores on HCV subjects (24) are related to psychological stress conditions, like obsessivecompulsive, phobic anxiety, paranoid ideation, psychoticism and lower scores on fighting spirit, hopelessness and anxious preoccupation towards illness than HIV-positive patients. Assessment of psychosocial variables and coping mechanisms should be integrated into all HCV and HIV services, especially those dedicated to the treatment of patients with substance abuse, as a vulnerable segment of the population at risk for life-threatening physical illness such as HCV and HIV infections.

There is evidence that immune system is responsible for an IFN- $\alpha$ depression, due to the fact that many depressive symptoms share features of somatic and behavioral symptoms, called "sickness behavior" (25).

Patients exposed to cytokines show a depressed level of functioning, which may allow the body to devote more energy to fighting illness. IFN- $\alpha$ stimulates the immune system, which can lead to in- 
creases of interleukin, which in turn have been correlated with higher depression scores.

Another research (26) found that subjects with an active psychiatric co-morbidity had significantly higher GSI and subscale T-scores compared to subjects with active medical co-morbidities and subjects without medical or psychiatric co-morbidities.

An Italian study (27) provides evidence between chronic hepatitis $\mathrm{C}$ and major depressive disorders. The study findings suggest that anxiety disorders are not a specific consequence of $\mathrm{HCV}$, but rather reflect the stress associated with the awareness of a chronic progressive disease or, in other patient series, alternative risk factors, such as drug or alcohol abuse.

A different view is provided by Kramer L (28). $\mathrm{He}$ concludes that patients with $\mathrm{HCV}$ infection showed slight but significant neurocognitive impairment, possibly indicating a further extrahepatic manifestation of chronic hepatitis $\mathrm{C}$.

\section{CONCLUSION}

There is a clear evidence and acceptance in the medical research and academia that HCV can impair the HRQOL of the patients. Very few studies until now have made an assessment of the patient's psychological condition at the moment when $\mathrm{HCV}$ infection was identified. Many researchers have established a link between the depression during and after the HCV treatment, but in most cases this is post HCV identification.

The extent of the impairment seems to vary being a function of various factors. The level of education, society development, substance abuse and awareness play an important role, as healthy individuals regard the $\mathrm{HCV}$ infected patients as normal individuals in Japan and as a burden (isolation, especially in women) in countries like Egypt or Saudi Arabia.

Patients unaware of the HCV infection seem to have a better HRQOL than those aware of the infection.

There is insufficient evidence at this time about the percentages of introverts/extroverts, depressive/non depressive patients at the moment of HCV positive identification. Further research is needed to accurately determine patient's psychological condition at the moment he is identified as $\mathrm{HCV}$ positive.

Evidence shows a direct correlation of the $\mathrm{HCV}$ with depression, but anxiety appears to be uncorrelated to any risk factors.

10. Dwight M.M., Kowdley K.V., Russo J.E., et al. Depression, fatigue, and functional disability in patients with chronic hepatitis $\mathrm{C}$. J Psychosom Res. 2000

11. Neda Svirtlih, Sladjana Pavic, Dragica Terzic, Dragan Delic, Jasmina Simonovic, Eleonora Gvozdenovic, Ivan Boricic, Reduced Quality of Life in Patients with Chronic Viral Liver Disease as Assessed by SF12 Questionnaire, Medical Faculty University of Belgrade, 2008

12. Ware J.E. Jr, Bayliss M.S., Mannocchia M., Davis G.L. Health related quality of life in chronic hepatitis $\mathrm{C}$ : Impact of disease and treatment response. The Interventional Therapy Group. Hepatology 1999; 30:550-5.

13. Shakil A.O., Conry-Cantilena C., Alter H.J., Hayashi P., Kleiner D.E., Tedeschi V., et al. Volunteer blood donors with antibody to hepatitis $C$ virus: Clinical, biochemical, virologic, and histologic features. The Hepatitis C Study Group. Ann Intern Med 1995; 123:330-7.

14. Fukuhara S., Hino K., Tomita E., Yuasa S., Okushin H. Healthrelated QOL in patients with chronic liver disease type-C (in Japanese with English abstract). Acta Hepatol Jpn 1997; 38:587-95

15. Ayman A. Abdo, Health-related quality of life of Saudi hepatitis B and C patients, King Saud University, Riyadh, 2012

16. Schwarzinger M., Dewedar S., Rekacewicz C., AbdElaziz K., Fontanet A., Carrat F., et al. Chronic hepatitis $C$ virus infection: Does it really impact health related quality of life? A study in rural Egypt. Hepatology 2004;

17. Golden J., O'Dwyer A.M., Conroy R.M. Depression and anxiety in patients with hepatitis C: prevalence, detection rates and risk factors, 2006 
18. Dwight M.M., Kowdley K.V., Russo J.E., Ciechanowski P.S. Larson A.M., Katon W.J. Depression, fatigue, and functional disability in patients with chronic hepatitis C. J Psychosom Res. 2000

19. Sockalingam S., Abbey S.E. Managing depression during hepatitis $C$ treatment. Can J Psychiatry. 2009

20. Hjerrild S., Renvillard S.G., Leutscher P.D., Videbech P. Increased prevalence of depression in hepatitis $C$ infection patients, Center for Psykiatrisk Forskning, Ugeskr Laeger, 2010

21. Elshahawi H., Hussein M., Allam A. Depression comorbidity in patients with chronic hepatitis $C$ and its possible relation to treatment outcome. Middle East Current Psychiatry, 2011

22. Patterson A.L., Morasco B.J., Fuller B.E., et al. Screening for depression in patients with hepatitis $C$ using the Beck Depression Inventory-II: do somatic symptoms compromise validity? Gen Hosp Psychiatry. 2011

23. Lehman C., Cheung R. Depression, anxiety, post-traumatic stress, and alcohol-related problems among veterans with chronic hepatitis C. Am J Gastroenterol. 2002
24. Grassi L., Satriano J., Serra A., Biancosino B., Zotos S., Sighinolfi L., Ghinelli F. Emotional stress, psychosocial variables and coping associated with hepatitis $C$ virus and human immunodeficiency virus infections in intravenous drug users, 2002

25. Smith K.J., Norris S., O'Farrelly C., et al. Risk factors for the development of depression in patients with hepatitis $\mathrm{C}$ taking interferon- $\alpha$. Neuropsychiatr Dis Treat. 2011

26. Fontana R., Hussain K., Schwartz S., Moyer C., Su G., Lok A. Emotional distress in chronic hepatitis $\mathrm{C}$ patients not receiving antiviral therapy. J Hepatol. 2002

27. Mauro G. Carta, Maria Carolina Hardoy, Alessandra Garofalo Enrica Pisano, Valentina Nonnoi, et al. Association of chronic hepatitis $\mathrm{C}$ with major depressive disorders: irrespective of interferonalpha therapy, 2007

28. Kramer L., Bauer E., Funk G., Hofer H., Jessner W., SteindlMunda P., Wrba F., Madl C., Gangl A., Ferenci P. Subclinical impairment of brain function in chronic hepatitis $\mathrm{C}$ infection. $\mathrm{J}$ Hepatol. 2002 